\title{
Suboptimal Event-Triggered Consensus of Multiagent Systems
}

\author{
Yuan Fan \\ School of Electrical Engineering and Automation, Anhui University, 111 Kowloon Road, Hefei 230601, China \\ Correspondence should be addressed to Yuan Fan; yuanf@mail.ustc.edu.cn
}

Received 5 August 2013; Accepted 26 February 2014; Published 23 March 2014

Academic Editor: Shawn X. Wang

Copyright (c) 2014 Yuan Fan. This is an open access article distributed under the Creative Commons Attribution License, which permits unrestricted use, distribution, and reproduction in any medium, provided the original work is properly cited.

\begin{abstract}
In this paper the suboptimal event-triggered consensus problem of Multiagent systems is investigated. Using the combinational measurement approach, each agent only updates its control input at its own event time instants. Thus the total number of events and the amount of controller updates can be significantly reduced in practice. Then, based on the observation of increasing the consensus rate and reducing the number of triggering events, we have proposed the time-average cost of the agent system and developed a suboptimal approach to determine the triggering condition. The effectiveness of the proposed strategy is illustrated by numerical examples.
\end{abstract}

\section{Introduction}

In practical application of networked dynamical systems, individual subsystems such as robots, vehicles, or mobile sensors are required to work cooperatively to accomplish complex tasks. This motivates the research on analysis and synthesis of networked dynamical systems and distributed coordination control of multiagent systems in recent years. Typical research directions in this filed include, but are not limited to, the problems of networked systems with unreliable communication links and quantized measurements [1-3], multiagent consensus [4-6], distributed tracking [7], formation control [8], connectivity preservation [9], agent flocking $[10-12]$, rendezvous $[13,14]$, coverage, and deployment [1517].

To reduce the total cost in practical systems, when implementing the communication and controller actuation schemes for multiagent systems, a possible design may equip each agent with a small embedded microprocessor and simple communication and actuation modules. However, these lowcost processors and modules usually have only limited energy and abilities. As a result, event-triggered schemes for practical control systems with digital platforms are proposed; see [1821]. Recently, event-based distributed control strategies have also been proposed for multiagent systems in [22-25]. Using the deterministic strategy introduced in [19], the control input of each agent is updated only when the measurement error magnitude exceeds a certain threshold. It is also proved that the lower bounds for the inter-event time intervals are strictly positive to ensure there is no Zeno behavior. Other works regarding event-triggered control for multiagent systems include decentralized control over wireless sensor networks [26], event-triggered consensus with second-order dynamics [27], and event-based leader-follower tracking [28].

The motivation of employing event-triggered control in multiagent systems is to reduce the costs of communication and controller updates so as to meet the hardware limitations and to save energy. However, in the existing event-triggered control of multiagent systems, the performance of the eventtriggered controller has not been studied yet [22-25]. In this paper, we firstly present a short review of the combinational measurement approach proposed in [29]. Compared with the existing approach, this approach allows the controller of each agent to be triggered only at the event time of itself, which reduces the frequency of controller updates in practice. Then, based on this control approach, we have investigated the optimal control problem of the event-triggered control system by formulating the average cost of the system. It is noted that the combinational measurement approach can be utilized to decouple the costs of different agents. By such cost decoupling strategy, a suboptimal approach to determine the 
TABLE 1: Average cost $J(g)$ with different feedback gains and cost coupling strengths; $\Delta=0.01$.

\begin{tabular}{lccc}
\hline$\beta_{i}$ & & $\left(\lambda_{i}, \xi_{i}\right)$ & \\
\hline 0.15 & $(0.7,0.2)$ & $(0.6,0.6)$ & $(0.1,0.8)$ \\
$\sqrt{2 \lambda_{i} \xi_{i}}$ & 2.6352 & 6.9189 & 1.5428 \\
0.90 & 2.0066 & 4.2239 & 1.4984 \\
\hline
\end{tabular}

triggering condition has been proposed. Numerical examples show that the proposed approach can reduce the total cost of the agent system during the consensus tasks.

The contribution of this work is as follows. Firstly, we have proposed a formulation of the time-average cost for multiagent systems with event-based controllers. This cost can describe the tradeoff between increasing the consensus rate and reducing the resource consumption. To the best of our knowledge, there has been very few works regarding this issue of event-triggered multiagent systems so far. Secondly, we have decoupled the costs of different agents and then found an upper bound of the cost for each agent. By this approach, we are able to propose a distributed suboptimal controller for the multiagent consensus problem.

The rest of this paper is organized as follows. Section 2 presents the event-triggered controller design of the multiagent system and the results of its convergence. In Section 3, the average cost of the system is formulated and the suboptimal triggering condition is obtained. In Section 4, simulations are provided to illustrate the proposed strategies. Finally the paper is concluded in Section 5.

\section{Event-Triggered Consensus}

In this section we provide a review of the event-triggered control with combinational measurement proposed in [29]. Consider a multiagent system with $N$ agents, labeled by $1,2, \ldots, N$, which are required to achieve the consensus task. The agent states at time $t$ are represented by $x_{i}(t) \in \mathbb{R}^{n}, i=$ $1, \ldots, N$. The dynamic of agent $i$ is

$$
\dot{x}_{i}(t)=u_{i}(t) .
$$

The communication links among agents are considered to be undirected and the communication topology of the system is represented by an undirected graph $G=(V, E)$, where $V$ is the vertex set and $E$ is the edge set. Agent $j$ is said to be a neighbor of agent $i$ if and only if $(j, i) \in E$ (or $(i, j) \in E$ ). All the neighbors of agent $i$ constitute the neighbor set $N_{i}$.

The event-triggering mechanism is introduced in agent control. The control input of agent $i$ will remain fixed until the next triggering event occurs. Assume that the triggering time sequence for agent $i$ is $t_{0}^{i}, t_{1}^{i}, \ldots, t_{k}^{i}, \ldots$, where $t_{0}^{i}=0$ is always a default triggering time. In the agent group, each agent can obtain the state information of its communication neighbors. When $t \in\left[t_{k}^{i}, t_{k+1}^{i}\right)$, the control input of agent $i$ will depend on the states of itself and its neighbors at time $t_{k}^{i}$.

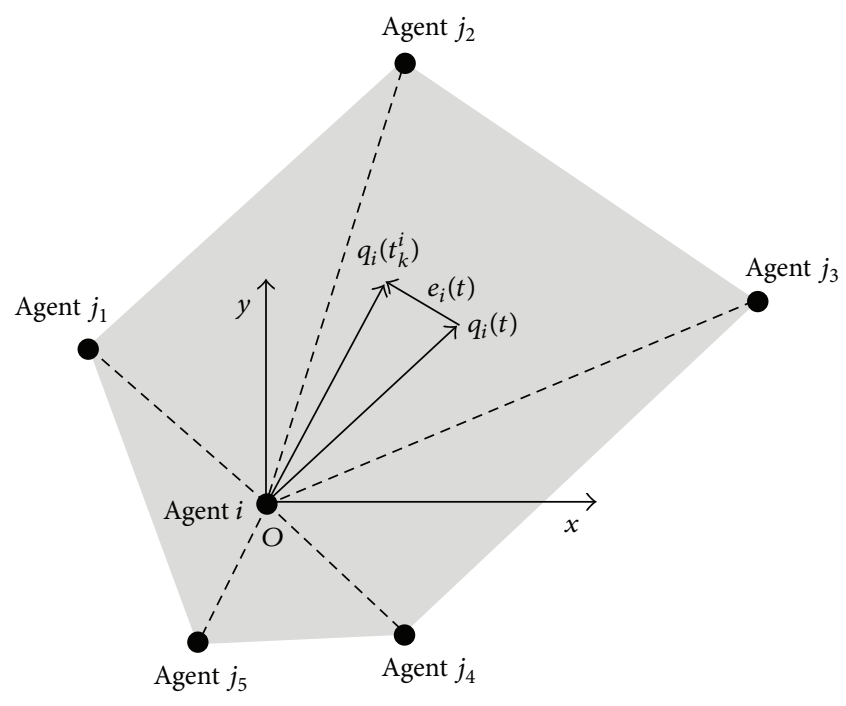

FIgURE 1: Target state and measurement error of agent $i$.

To develop decentralized control, agent $i$ 's local coordinate system is introduced and the origin is at $x_{i}(t)$. The realtime average state of agent $i$ and all its neighbors in this local coordinate system is

$$
q_{i}(t)=\frac{1}{n_{i}+1} \sum_{j \in N_{i}}\left(x_{j}(t)-x_{i}(t)\right) .
$$

At each triggering time point, agent $i$ measures this average state and takes the measurement as its target; see Figure 1 for an illustration in a 2D plane. This target state will remain fixed until the next triggering time comes. Thus the target state of agent $i$ when $t \in\left[t_{k}^{i}, t_{k+1}^{i}\right)$ is

$$
q_{i}\left(t_{k}^{i}\right)=\frac{1}{n_{i}+1} \sum_{j \in N_{i}}\left(x_{j}\left(t_{k}^{i}\right)-x_{i}\left(t_{k}^{i}\right)\right) .
$$

For $t \in\left[t_{k}^{i}, t_{k+1}^{i}\right)$, the control law for agent $i$ is proposed in [29] as follows:

$$
\begin{aligned}
u_{i}(t) & =\xi_{i} q_{i}\left(t_{k}^{i}\right) \\
& =\frac{\xi_{i}}{n_{i}+1} \sum_{j \in N_{i}}\left(x_{j}\left(t_{k}^{i}\right)-x_{i}\left(t_{k}^{i}\right)\right),
\end{aligned}
$$

where $\xi_{i}$ is a positive real number to be determined. The measurement error of agent $i$ will be

$$
e_{i}(t)=q_{i}\left(t_{k}^{i}\right)-q_{i}(t)
$$

Since $t_{0}^{i}=0$ is a triggering time instant, one has $e_{i}(0)=0$. In the sequel we will show how to use this error to determine the triggering event that guarantees the consensus of the agent group.

We denote $x(t)=\left(x_{1}^{T}(t), \ldots, x_{N}^{T}(t)\right)^{T}$ as the augmented state of the system and also denote $e(t)=\left(e_{1}^{T}(t), \ldots, e_{N}^{T}(t)\right)^{T}$. From (4) and (5) one has

$$
\dot{x}_{i}(t)=-\frac{\xi_{i}}{n_{i}+1} \sum_{j \in N_{i}}\left(x_{i}(t)-x_{j}(t)\right)+\xi_{i} e_{i}(t) .
$$



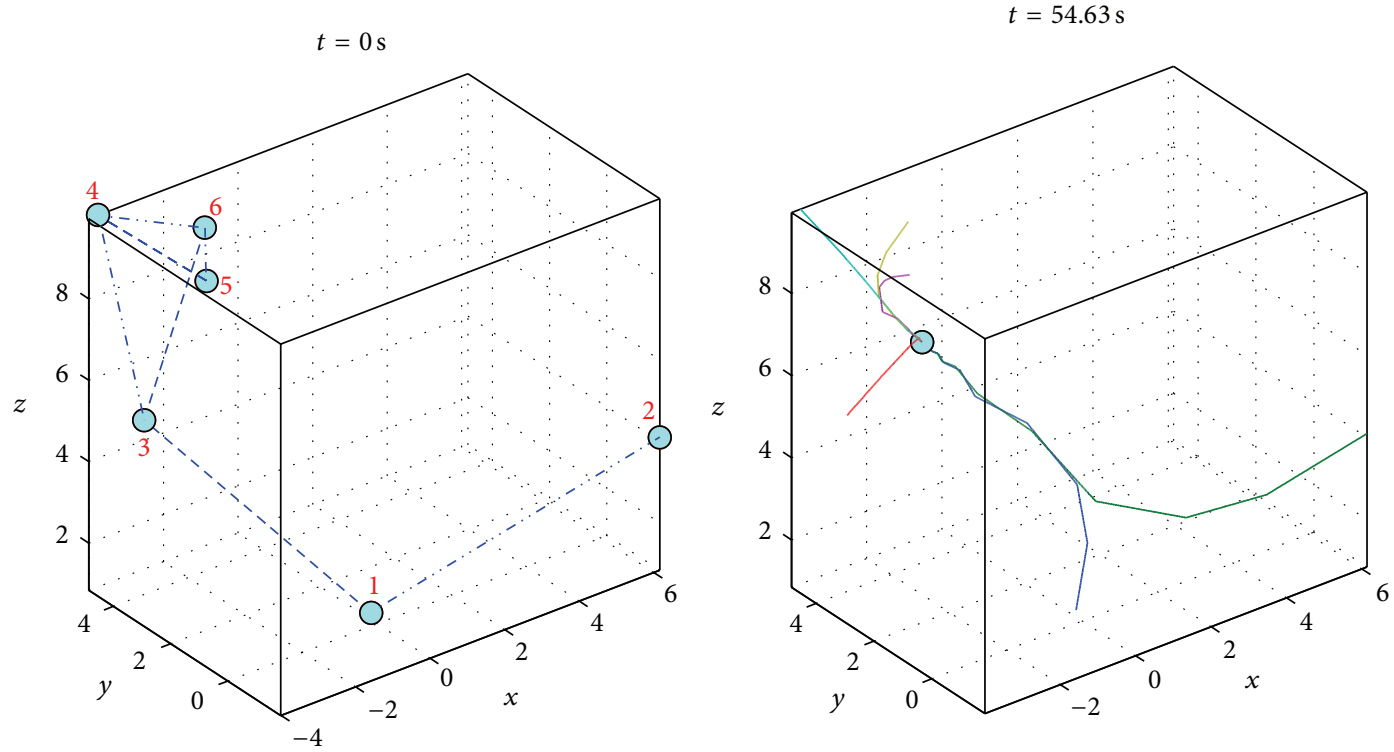

Figure 2: Communication topology and trajectories of agents.
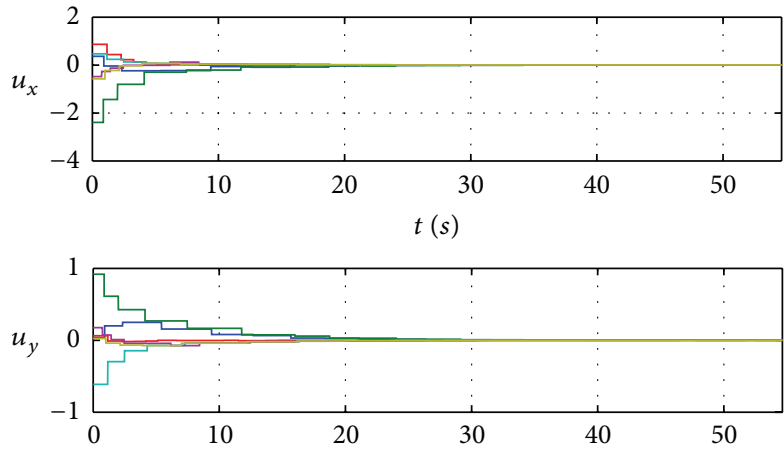

$t(s)$

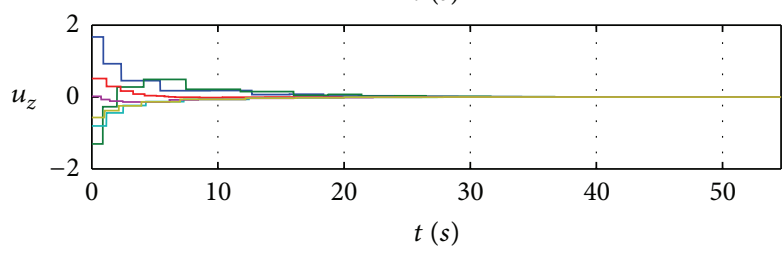

Figure 3: Control inputs of agents.

Let $L$ be the Laplacian matrix of the underlying graph $G$. Also let $\Xi=\operatorname{diag}\left\{\xi_{1}, \ldots, \xi_{N}\right\}$ and $M=\operatorname{diag}\left\{n_{1}+1, \ldots, n_{N}+1\right\}$. Then the compact form of the system equation is given by

$$
\dot{x}(t)=-\left((\Lambda L) \otimes I_{n}\right) x(t)+\left(\Xi \otimes I_{n}\right) e(t),
$$

where $\Lambda=M^{-1} \Xi$. Since the communication is bidirectional, graph $G$ is undirected and then $L$ is symmetric [5]. Consider the candidate Lyapunov function

$$
\mathbf{V}(t)=\frac{1}{2} x^{T}(t)\left(L \otimes I_{n}\right) x(t) .
$$

One has

$$
\begin{aligned}
\dot{\mathbf{V}}(t) & =x^{T}(t)\left(L \otimes I_{n}\right) \dot{x}(t) \\
& =x^{T}(t)\left(L \otimes I_{n}\right)\left(-\left((\Lambda L) \otimes I_{n}\right) x(t)+\left(\Xi \otimes I_{n}\right) e(t)\right) .
\end{aligned}
$$

Let $z_{i}(t)=\sum_{j \in N_{i}}\left(x_{i}(t)-x_{j}(t)\right)$ and

$$
z(t)=\left(z_{1}^{T}(t), \ldots, z_{N}^{T}(t)\right)^{T} .
$$

Then one has

$$
z(t)=\left(L \otimes I_{n}\right) x(t) .
$$

From (9),

$$
\begin{aligned}
\dot{\mathbf{V}}(t) & =-z^{T}(t)\left(\Lambda \otimes I_{n}\right) z(t)+z^{T}(t)\left(\Xi \otimes I_{n}\right) e(t) \\
& =-\sum_{i=1}^{N} \frac{\xi_{i}}{n_{i}+1}\left\|z_{i}(t)\right\|^{2}+\sum_{i=1}^{N} \xi_{i} z_{i}^{T}(t) e_{i}(t) .
\end{aligned}
$$

Note that for any $a>0$ and any $x, y \in \mathbb{R}^{n}$, one always has $\left|x^{T} y\right| \leq(a / 2)\|x\|^{2}+(1 / 2 a)\|y\|^{2}$. Thus

$$
\begin{aligned}
\dot{\mathbf{V}}(t) \leq & -\sum_{i=1}^{N} \frac{\xi_{i}}{n_{i}+1}\left\|z_{i}(t)\right\|^{2} \\
& +\sum_{i=1}^{N} \frac{a_{i} \xi_{i}}{2}\left\|z_{i}(t)\right\|^{2} \\
& +\sum_{i=1}^{N} \frac{\xi_{i}}{2 a_{i}}\left\|e_{i}(t)\right\|^{2} .
\end{aligned}
$$

Enforcing

$$
\left\|e_{i}(t)\right\| \leq \eta_{i}\left\|z_{i}(t)\right\|
$$



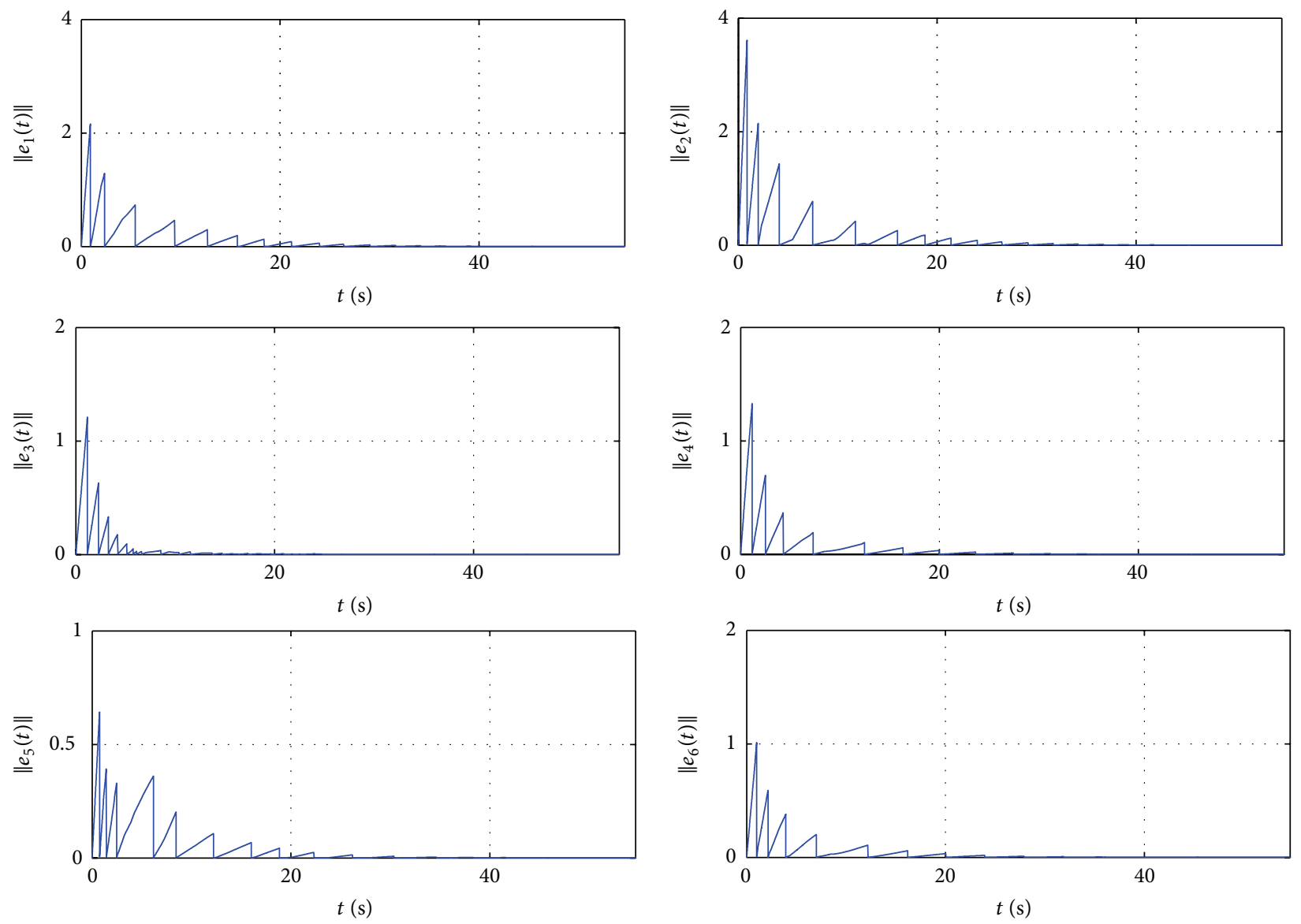

FIGURE 4: Measurement error norms of agents.

yields

$$
\dot{\mathbf{V}}(t) \leq-\sum_{i=1}^{N}\left(\frac{\xi_{i}}{n_{i}+1}-\frac{a_{i} \xi_{i}}{2}-\frac{\xi_{i} \eta_{i}^{2}}{2 a_{i}}\right)\left\|z_{i}(t)\right\|^{2}
$$

Thus $\dot{\mathbf{V}}(t) \leq 0$ if $1 /\left(n_{i}+1\right)-a_{i} / 2-\eta_{i}^{2} / 2 a_{i}>0$. From this, one has $a_{i}<2 /\left(n_{i}+1\right)$ and $\eta_{i}<\sqrt{2 a_{i} /\left(n_{i}+1\right)-a_{i}^{2}}$. Notice that, when $a_{i}=1 /\left(n_{i}+1\right), \sqrt{2 a_{i} /\left(n_{i}+1\right)-a_{i}^{2}}$ reaches its maximum $1 /\left(n_{i}+1\right)$. Also notice that

$$
z_{i}(t)=-\left(n_{i}+1\right) q_{i}(t)
$$

Thus (14) can be rewritten as

$$
\left\|e_{i}(t)\right\| \leq \beta_{i}\left\|q_{i}(t)\right\|, \quad 0<\beta_{i}<1 .
$$

Then (15) becomes

$$
\dot{\mathbf{V}}(t) \leq-\sum_{i=1}^{N} \frac{\xi_{i}\left(1-\beta_{i}^{2}\right)}{2\left(n_{i}+1\right)}\left\|z_{i}(t)\right\|^{2} .
$$

The triggering function for agent $i$ is

$$
g_{i}\left(e_{i}(t), q_{i}(t)\right)=\left\|e_{i}(t)\right\|-\beta_{i}\left\|q_{i}(t)\right\|, \quad 0<\beta_{i}<1 .
$$

And an event of agent $i$ will be triggered when

$$
g_{i}\left(e_{i}(t), q_{i}(t)\right)=0 \text {. }
$$

Notice that when an event is triggered, the control input changes and the error $e_{i}(t)$ is automatically reset to 0 .

It is noted that the triggering mechanism is designed in such a way that the time derivative of the Lyapunov function is enforced to be nonpositive by (17). However, this does not sufficiently guarantee the convergence of the closed-loop system. In a hybrid system, the interevent time may get shorter and shorter for increasing $k$ such that infinitely many events are triggered in a finite time interval. Such execution of a hybrid system is called Zeno; see [30] and references therein for more details. Generally speaking, in controller design one may expect the agents are always triggered regularly and there is no Zeno behavior. Actually, in [29] comprehensive triggering behavior analysis has been provided and we have the following lemma.

Lemma 1. Consider an agent $i$ with a nonempty neighbor set $N_{i}$. Its kinematic is given in (1) and its controller is the eventtriggered control (4), with (20) being the triggering condition. If $t_{k}^{i}$ exists and $q_{i}\left(t_{k}^{i}\right) \neq 0$, agent $i$ will only exhibit regular triggering behavior for all $t>t_{k}^{i}$. 

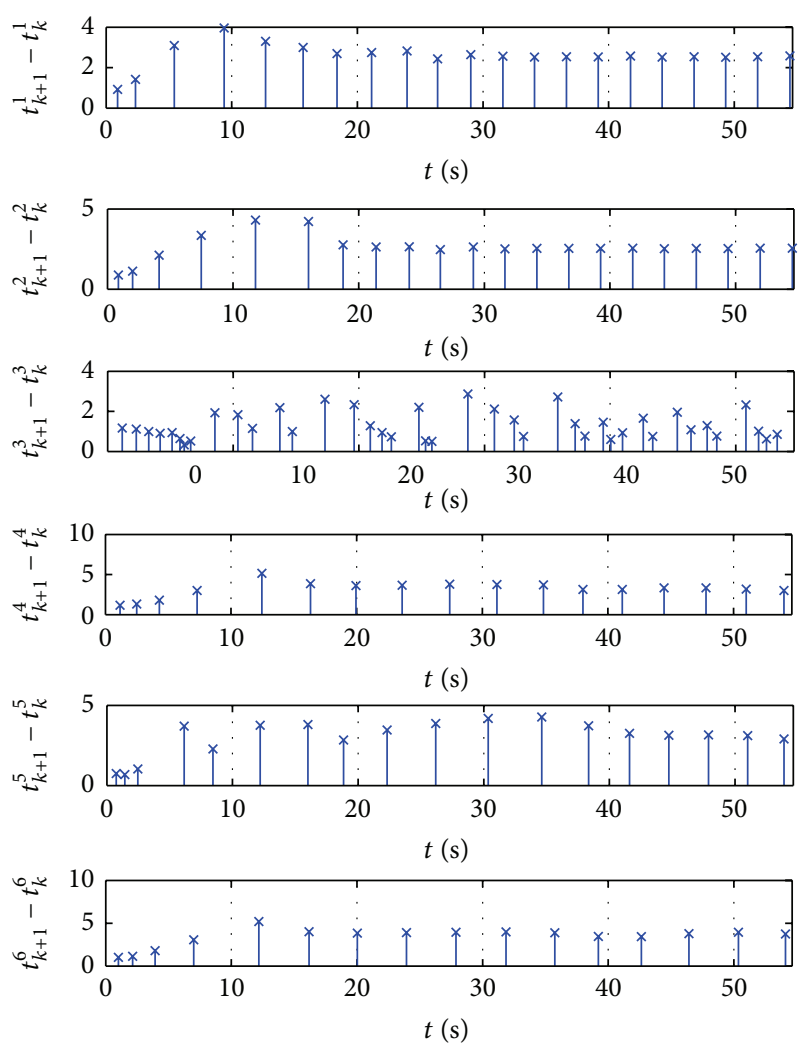

FIgURE 5: Event instants and time intervals of agents.

Proof. The proof of this lemma follows directly from Lemmas 2,3 , and 4 in [29].

Then we are at the position to present the consensus result of the proposed event-triggered controller.

Theorem 2 (see [29]). Consider a group of $N$ agents moving in the working space $\mathbb{R}^{n}$. The dynamic of each agent is (1). Assume that the communication graph $G$ is fixed and connected. If no agent is located at the average state of its neighbors, the group will achieve consensus asymptotically under the event-triggered control law (4) with the triggering condition (20).

Remark 3. We note that the agent group may not achieve consensus if more than one agent is located at the average of all its neighbors. One strategy for solving this problem is to use a subset of $N_{i}$; for example, $N_{i}^{s} \subset N_{i}$, to compute $q_{i}(k+1)$ and $t_{k+1}^{i}$. Then, at $t_{k+1}^{i}$, when agent $i$ is no longer at the average state of all its neighbors, the controller is switched back to use $N_{i}$.

\section{Suboptimal Triggering}

In a practical multiagent system, fast achievement of the coordination tasks with least resource consumption is often expected. For consensus problem discussed in this work, one may expect the highest consensus rate with the least amount of events and controller updating executions. However, there is a tradeoff between these two factors. On the one hand, to achieve fast consensus and precise control, one may require the norm of the measurement error $\left\|e_{i}(t)\right\|$ as smaller as possible, which may call for high frequency of event triggering and controller updating. On the other hand, to save energy and communication bandwidth, one may reduce the triggering frequency and thus the amount of controller updating, which contradicts the above mentioned consensus rate expectation. The goal of this section is to balance a tradeoff between increasing the consensus rate and reducing events and controller updates.

It is noted that, if $\left\|e_{i}(t)\right\|=0$, agent $i$ takes the center of all its neighbors $q_{i}(t)$ as the target point. Then it may achieve consensus faster than using $q_{i}\left(t_{k}^{i}\right)$ since $q_{i}(t)$ is the real-time neighborhood center. Thus $\left\|e_{i}(t)\right\|$ can be considered as the measurement cost of agent $i$. The smaller this cost is, the faster the consensus rate can be. However, directly using $\left\|e_{i}(t)\right\|$ as the measurement cost is not a better choice since, when all the agents are very close to each other, $\left\|e_{i}(t)\right\|$ goes to 0 and cannot reflect the consensus rate well. To solve this problem, we let the measurement cost of agent $i$ be

$$
s_{i}(t)=\frac{\left\|e_{i}(t)\right\|}{\left\|q_{i}(t)\right\|} .
$$

This definition can represent the measurement deviation of the real-time neighborhood center from its true value. It is better than directly using $\left\|e_{i}(t)\right\|$ as the measurement cost since $s_{i}(t)$ is also well defined to reflect the consensus rate when $\left\|e_{i}(t)\right\|$ tends to 0 as time goes to infinity. The lower this cost is, the faster the consensus rate can be.

To formulate the above mentioned tradeoff, we should also find a way to count the amount of triggering events for all the agents. Let the entire triggering cost of agent $i$ be its total number of triggering events. Then during the time interval $\left[t_{k}^{i}, t_{k+1}^{i}\right)$, the triggering cost of agent $i$ is 1 . Thus we can define the time-average triggering cost as

$$
\sigma_{i}(t)=\frac{2}{\left(t_{k+1}^{i}-t_{k}^{i}\right)^{2}}\left(t-t_{k}^{i}\right), \quad t \in\left[t_{k}^{i}, t_{k+1}^{i}\right) .
$$

This definition implies that the total cost in a single event time interval $\left[t_{k}^{i}, t_{k+1}^{i}\right)$ is always 1 ; that is,

$$
\int_{t_{k}^{i}}^{t_{k+1}^{i}} \sigma_{i}(t) d t=1
$$

The lower the cost $\sigma_{i}(t)$, the fewer the amount of event triggering and controller updating and thus the resource consumption can be.

Then we can define the comprehensive time-average cost of agent $i$, that is, the per-period cost, as

$$
s_{i}(t)+\lambda_{i} \sigma_{i}(t)
$$

where $\lambda_{i}$ is the cost coupling strength. The objective is to find a balance between the estimation error and the triggering frequency. Namely, we are aiming to find a set of optimal 
triggering policies to minimize the average cost of the agent group, which is defined by

$$
J(g)=\lim _{T \rightarrow \infty} \frac{1}{T} \int_{0}^{T} \sum_{i=1}^{N}\left(s_{i}(t)+\lambda_{i} \sigma_{i}(t)\right) d t,
$$

where $g=\left\{g_{i}, \ldots, g_{N}\right\}$ is the collection of triggering functions. Since all the agents are coupled by the eventtriggered control, the whole group exhibits the behavior of a complex hybrid system. Thus to solve the problem of minimizing $J(g)$ by designing $g$ is rather challenging. However, based on the behavior analysis presented in [29], one can find a suboptimal solution to this problem.

Denote $J(g)=\sum_{i=1}^{N} J_{i}(g)$ where

$$
J_{i}(g)=\lim _{T \rightarrow \infty} \frac{1}{T} \int_{0}^{T}\left(s_{i}(t)+\lambda_{i} \sigma_{i}(t)\right) d t
$$

is the average cost of agent $i$. Let $t_{p}$ and $t_{q}$ be two event instants of agent $i$ with $t_{q}>t_{p}$, and let

$$
T=t_{q}-t_{p}
$$

We consider this cost over a sufficiently long time period $[0, T]$. From (25) one notices that the finite time form of agent $i$ 's average cost over the time interval $\left[t_{p}, t_{q}\right]$ is

$$
\begin{aligned}
J_{i}\left[t_{p}, t_{q}\right] & =\frac{1}{T} \int_{t_{p}}^{t_{q}}\left(s_{i}(t)+\lambda_{i} \sigma_{i}(t)\right) d t \\
& =\frac{1}{T} \sum_{k=p}^{q-1} \int_{t_{k-1}^{i}}^{t_{k}^{i}}\left(s_{i}(t)+\lambda_{i} \sigma_{i}(t)\right) d t \\
& =\frac{1}{T} \sum_{k=p}^{q-1} \int_{t_{k-1}^{i}}^{t_{k}^{i}} s_{i}(t) d t+\frac{\lambda_{i}}{T} \sum_{k=p}^{q-1} \int_{t_{k-1}^{i}}^{t_{k}^{i}} \sigma_{i}(t) d t .
\end{aligned}
$$

From (17) one has $s_{i}(t) \leq \beta_{i}$, and they are equal only when $t=t_{k}^{i}$. Then from (23) one has

$$
\begin{aligned}
J_{i}\left[t_{p}, t_{q}\right] & <\frac{\beta_{i}}{T} \sum_{k=p}^{q-1}\left(t_{k}^{i}-t_{k-1}^{i}\right)+\frac{\lambda_{i}}{T} \sum_{k=p}^{q-1} 1 \\
& =\beta_{i}+\frac{\lambda_{i} M}{T},
\end{aligned}
$$

with $M$ being the number of event time intervals on $\left[t_{p}, t_{q}\right]$. Thus, when $t_{q} \rightarrow \infty$, the average cost of agent $i$ can be upper bounded by

$$
J_{i}\left[t_{p}, t_{q}\right]<\beta_{i}+\frac{\lambda_{i}}{\tau_{i}},
$$

where $\tau_{i}=T / M$ is the average length of triggering time interval of agent $i$. It is difficult to obtain an estimation of $\tau_{i}$. However, one may consider the average cost of agent $i$ on the time interval $\left[t_{p}, t_{q}\right]$ when $t_{p}$ is sufficiently large. In this case, $\tau_{i}$ will be lower bounded by $\tau_{k}^{i}$, s limit defined in [29]. Consider

$$
\lim _{k \rightarrow \infty} \tau_{k}^{i}=\frac{\beta_{i}}{2 \zeta_{i}\left(1+\beta_{i}\right)}
$$

where $\zeta_{i}=\max _{l}\left\{\xi_{l} \mid l \in N_{i} \cup\{i\}\right\}$. Thus one has

$$
\begin{aligned}
J_{i}\left[t_{p}, t_{q}\right] & <\beta_{i}+\frac{\lambda_{i}}{\beta_{i} /\left(2 \zeta_{i}\left(1+\beta_{i}\right)\right)} \\
& =2 \lambda_{i} \zeta_{i}+\beta_{i}+\frac{2 \lambda_{i} \zeta_{i}}{\beta_{i}} .
\end{aligned}
$$

The right-hand side will reach its minimum if and only if $\beta_{i}=$ $\sqrt{2 \lambda_{i} \zeta_{i}}$. If all agents take the same $\xi_{i}$, this condition will be

$$
\beta_{i}=\sqrt{2 \lambda_{i} \xi_{i}}
$$

Thus a suboptimal triggering condition is given by

$$
g_{i}\left(\left\|e_{i}(t), q_{i}(t)\right\|\right)=\left\|e_{i}(t)\right\|-\sqrt{2 \lambda_{i} \xi_{i}}\left\|q_{i}(t)\right\|=0
$$

if $\sqrt{2 \lambda_{i} \xi_{i}}<1$

Remark 4. Equation (33) shows the relationship between the triggering execution and the importance of the measurement and triggering cost. For example, a larger $\lambda_{i}$ means reducing the triggering cost is more important. Then one will obtain a larger $\beta_{i}$, which may lengthen the time in between consecutive triggering executions and reduce the triggering cost.

\section{Simulations}

In this section some simulations will be provided to illustrate the proposed event-triggered control strategy. Consider a group of $N=6$ agents in the working space $\mathbb{R}^{3}$. Each agent has dynamic (1) and the controller (4). The parameters in the control input (4) and the triggering function (19) are given by $\xi_{i}=0.4$ and $\beta_{i}=0.9$ for all agents. The initial states of agents are randomly selected which are as follows:

$$
\begin{aligned}
& x_{1}(0)=(0.1725,0.4469,0.8357)^{T}, \\
& x_{2}(0)=(6.1630,-1.8492,4.1066)^{T}, \\
& x_{3}(0)=(-4.0025,2.9335,5.9033)^{T}, \\
& x_{4}(0)=(-3.7577,4.8591,9.9656)^{T}, \\
& x_{5}(0)=(-1.9829,3.3818,8.4150)^{T}, \\
& x_{6}(0)=(-1.8028,3.6658,9.5204)^{T} .
\end{aligned}
$$

The communication graph $G$ is also shown in the first subfigure of Figure 2. In the simulation, when the sum of the distances from agents to the group average is shorter than $\Delta=0.01$; that is, $\sum_{i=1}^{N}\left\|x_{i}(t)-(1 / N) \sum_{i=1}^{N} x_{i}(t)\right\| \leq \Delta$, the group is considered to have achieved consensus.

The trajectories of agents in the simulation are shown in Figure 2. The agents are represented by small circles and the trajectories are represented by solid lines. Notice that the agent group eventually achieve consensus at $t=54.63 \mathrm{~s}$ under the proposed control law. One can also note from the trajectories that the control inputs of all the agents, which 
are shown in Figure 3, are fixed during each interevent time interval.

Figure 4 shows the evolution of the error norms of all the agents. From (20) one concludes that the curve of these error norms stays below the threshold $\beta_{i}\left\|q_{i}(t)\right\|$. The error increases in each triggering time interval and then is automatically reset to 0 when an event occurs.

The event time instants of all the agents are shown in Figure 5. From this figure one can observe that all agents are triggered regularly and the interevent time intervals have strictly positive lengths. This implies there is no Zeno behavior in the system evolution. Moreover, the input of each agent only triggers when its own event occurs.

To verify the proposed suboptimal triggering approach, a set of similar simulations are carried out with different parameter selections. The initial conditions of the agents are the same as in Figure 2. The results are listed in Table 1. The table shows that, following the proposed strategy, the cost can be obviously reduced compared with those under the choices which appear appropriate. Simulations also show that, in some cases, the suboptimal choices are very close to the optimal ones.

\section{Conclusions}

In this paper, the suboptimal event-triggered consensus problem for multiagent systems is considered. The event design is based on the measurement error which is determined by a combined state of neighbors. As a result, each agent only updates its controller at its own event time, which reduces the amount of interagent communication and controller updates in practice. Then we have proposed a novel definition of timeaverage cost for the agent system and developed a suboptimal triggering approach to determine the event condition. It has been shown that the proposed approach is effective in reducing the average cost of the system. Future work includes extending the proposed approach to multiagent systems with directed communication networks and developing better optimization approach to reduce the system cost.

\section{Conflict of Interests}

The author declares that there is no conflict of interests regarding the publication of this paper.

\section{Acknowledgments}

The work described in this paper was partially supported by grants from the National Natural Science Foundation of China (no. 61203027), the Specialized Research Fund for the Doctoral Program of Higher Education of China (no. 20123401120011), and the Anhui Provincial Natural Science Foundation (no. 1208085QF108).

\section{References}

[1] J. Qiu, G. Feng, and H. Gao, "Fuzzy-model-based piecewise Hinfinity static output feedback controller design for networked nonlinear systems," IEEE Transactions on Fuzzy Systems, vol. 18, no. 5, pp. 919-934, 2010.

[2] J. Qiu, G. Feng, and H. Gao, "Nonsynchronized-State estimation of multichannel networked nonlinear systems with multiple packet dropouts via T-S fuzzy-affine dynamic models," IEEE Transactions on Fuzzy Systems, vol. 19, no. 1, pp. 75-90, 2011.

[3] J. Qiu, G. Feng, and H. Gao, "Observer-based piecewise affine output feedback controller synthesis of continuous-time T-S fuzzy affine dynamic systems using quantized measurements," IEEE Transactions on Fuzzy Systems, vol. 20, no. 6, pp. 10461062, 2012.

[4] W. Ren and R. W. Beard, "Consensus seeking in multiagent systems under dynamically changing interaction topologies," IEEE Transactions on Automatic Control, vol. 50, no. 5, pp. 655661, 2005.

[5] R. Olfati-Saber, J. A. Fax, and R. M. Murray, "Consensus and cooperation in networked multi-agent systems," Proceedings of the IEEE, vol. 95, no. 1, pp. 215-233, 2007.

[6] L. Xiao, X. Liao, and H. Wang, "Cluster consensus on discretetime multi-agent networks," Abstract and Applied Analysis, vol. 2012, Article ID 274735, 11 pages, 2012.

[7] J. Hu and G. Feng, "Distributed tracking control of leaderfollower multi-agent systems under noisy measurement," Automatica, vol. 46, no. 8, pp. 1382-1387, 2010.

[8] J. A. Marshall and D. Tsai, "Periodic formations of multivehicle systems," IET Control Theory \& Applications, vol. 5, no. 2, pp. 389-396, 2011.

[9] Y. Fan, L. Liu, G. Feng, C. Song, and Y. Wang, "Virtual neighbor based connectivity preserving of multi-agent systems with bounded control inputs in the presence of unreliable communication links," Automatica, vol. 49, no. 5, pp. 1261-1267, 2013.

[10] M. M. Zavlanos, H. G. Tanner, A. Jadbabaie, and G. J. Pappas, "Hybrid control for connectivity preserving flocking," IEEE Transactions on Automatic Control, vol. 54, no. 12, pp. 28692875, 2009.

[11] Z. Chen and H.-T. Zhang, "No-beacon collective circular motion of jointly connected multi-agents," Automatica, vol. 47, no. 9, pp. 1929-1937, 2011.

[12] Z. Chen and H. T. Zhang, "Analysis of joint connectivity condition for multi-agents with boundary constraints," IEEE Transactions on Cybernetics, vol. 43, no. 2, pp. 437-444, 2013.

[13] J. Cortés, S. Martínez, and F. Bullo, "Robust rendezvous for mobile autonomous agents via proximity graphs in arbitrary dimensions," IEEE Transactions on Automatic Control, vol. 51, no. 8, pp. 1289-1298, 2006.

[14] Y. Fan, G. Feng, Y. Wang, and J. Qiu, "A novel approach to coordination of multiple robots with communication failures via proximity graph," Automatica, vol. 47, no. 8, pp. 1800-1805, 2011.

[15] C. Song, G. Feng, Y. Fan, and Y. Wang, "Decentralized adaptive awareness coverage control for multi-agent networks," Automatica, vol. 47, no. 12, pp. 2749-2756, 2011.

[16] C. Nowzari and J. Cortés, "Self-triggered coordination of robotic networks for optimal deployment," Automatica, vol. 48, no. 6, pp. 1077-1087, 2012.

[17] C. Song, L. Liu, G. Feng, Y. Wang, and Q. Gao, "Persistent awareness coverage control for mobile sensor networks," Automatica, vol. 49, no. 6, pp. 1867-1873, 2013.

[18] K. J. Åström and B. M. Bernhardsson, "Comparison of Riemann and Lebesgue sampling for first order stochastic systems," in 
Proceedings of the 41st IEEE Conference on Decision and Control, pp. 2011-2016, Las Vegas, Nev, USA, December 2002.

[19] P. Tabuada, "Event-triggered real-time scheduling of stabilizing control tasks," IEEE Transactions on Automatic Control, vol. 52, no. 9, pp. 1680-1685, 2007.

[20] M. Mazo Jr., A. Anta, and P. Tabuada, "An ISS self-triggered implementation of linear controllers," Automatica, vol. 46, no. 8, pp. 1310-1314, 2010.

[21] X. Wang and M. D. Lemmon, "Event-triggering in distributed networked control systems," IEEE Transactions on Automatic Control, vol. 56, no. 3, pp. 586-601, 2011.

[22] D. V. Dimarogonas and K. H. Johansson, "Event-triggered cooperative control," in Proceedings of the European Control Conference, pp. 3015-3020, Budapest, Hungary, August 2009.

[23] D. V. Dimarogonas and K. H. Johansson, "Event-triggered control for multi-agent systems," in Proceedings of the 48th IEEE Conference on Decision and Control Held Jointly with 28th Chinese Control Conference (CDC/CCC '09), pp. 7131-7136, Shanghai, China, December 2009.

[24] D. V. Dimarogonas, E. Frazzoli, and K. H. Johansson, "Distributed event-triggered control for multi-agent systems," IEEE Transactions on Automatic Control, vol. 57, no. 5, pp. 1291-1297, 2012.

[25] G. S. Seyboth, D. V. Dimarogonas, and K. H. Johansson, "Event-based broadcasting for multi-agent average consensus," Automatica, vol. 49, no. 1, pp. 245-252, 2013.

[26] M. Mazo Jr. and P. Tabuada, "Decentralized event-triggered control over wireless sensor/actuator networks," IEEE Transactions on Automatic Control, vol. 56, no. 10, pp. 2456-2461, 2011.

[27] J. Hu, Y. Zhou, and Y. Lin, "Second-order multiagent systems with event-driven consensus control," Abstract and Applied Analysis, vol. 2013, Article ID 250586, 9 pages, 2013.

[28] J. Hu, G. Chen, and H. X. Li, "Distributed event-triggered tracking control of leader-follower multi-agent systems with communication delays," Kybernetika, vol. 47, no. 4, pp. 630-643, 2011.

[29] Y. Fan, G. Feng, Y. Wang, and C. Song, "Distributed eventtriggered control of multi-agent systems with combinational measurements," Automatica, vol. 49, no. 2, pp. 671-675, 2013.

[30] J.Zhang, K. H. Johansson, J. Lygeros, and S. Sastry, "Zeno hybrid systems," International Journal of Robust and Nonlinear Control, vol. 11, no. 5, pp. 435-451, 2001. 


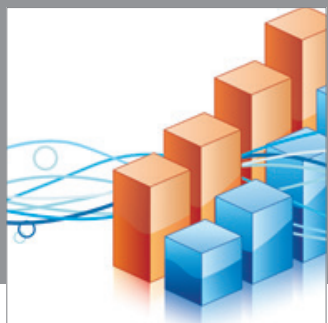

Advances in

Operations Research

mansans

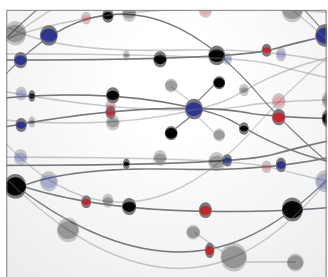

The Scientific World Journal
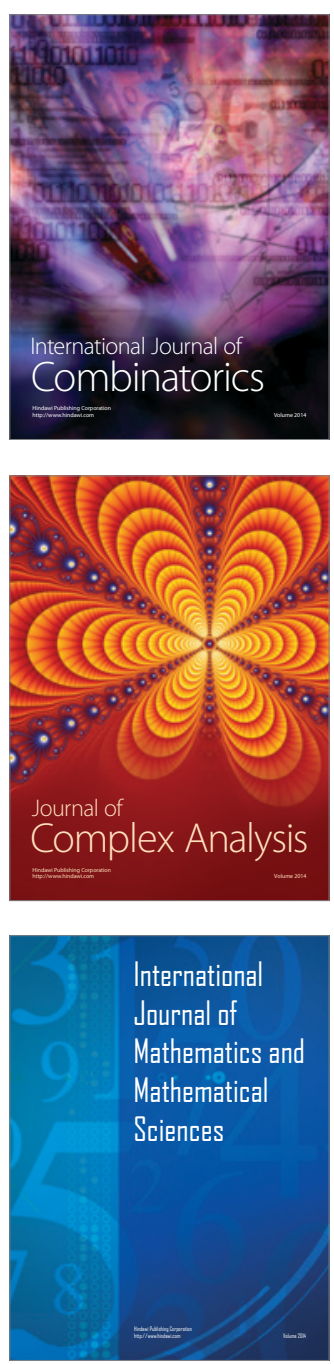
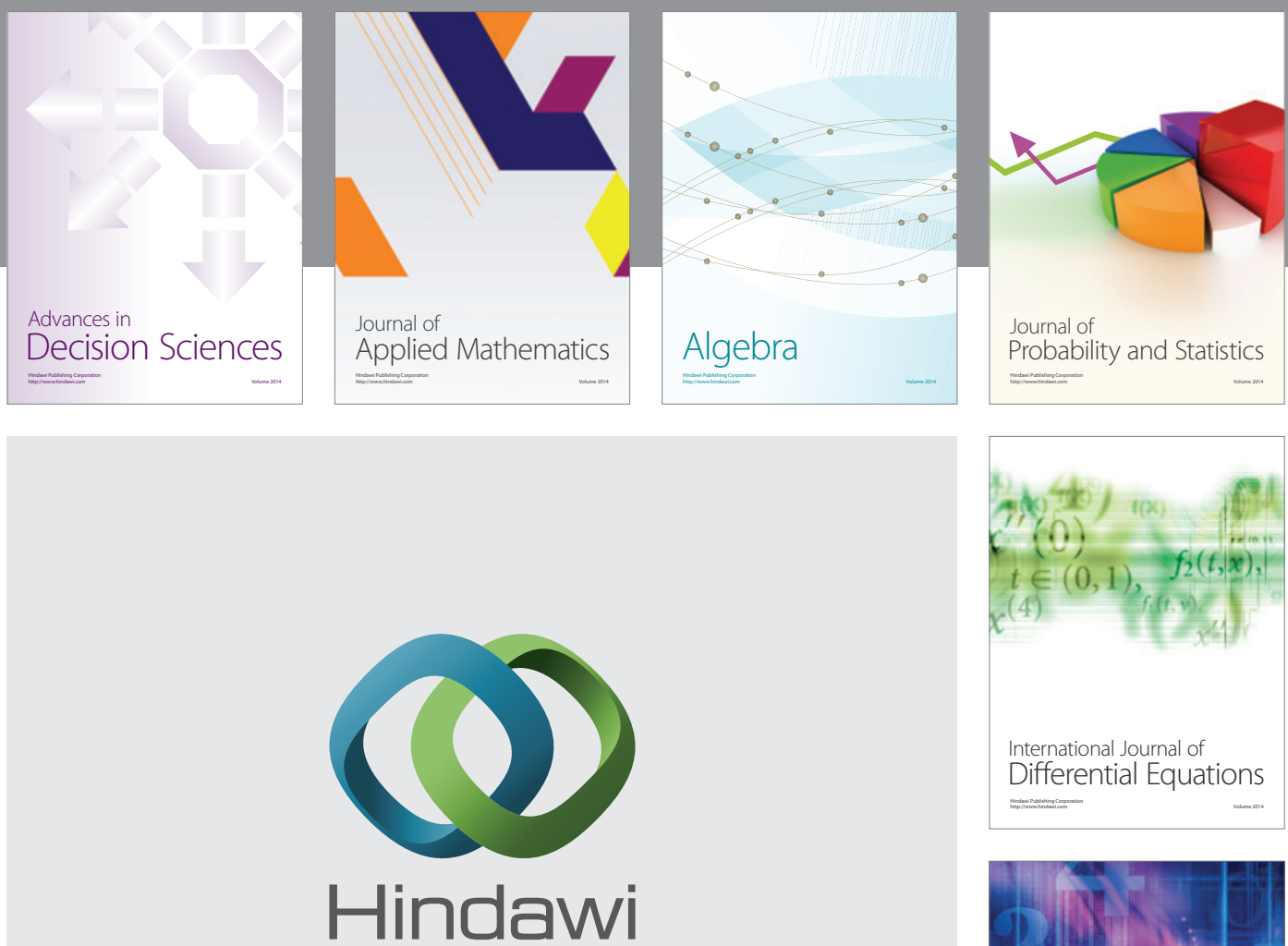

Submit your manuscripts at http://www.hindawi.com
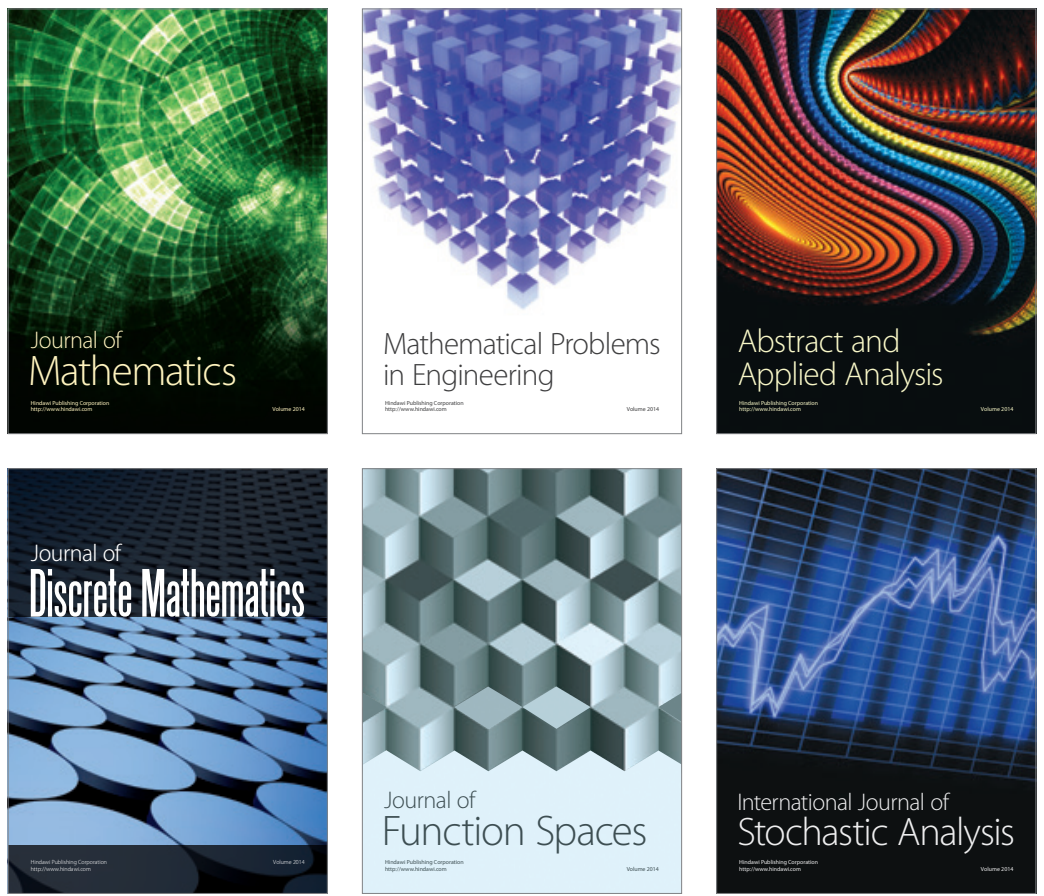

Journal of

Function Spaces

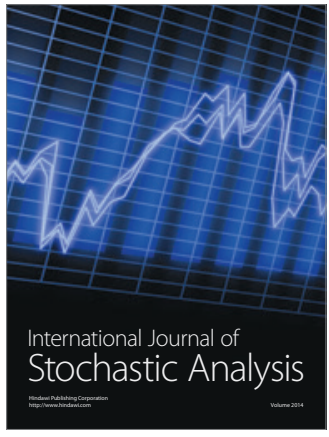

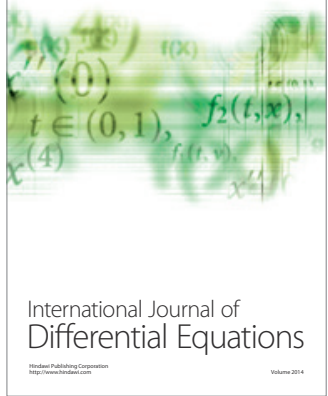
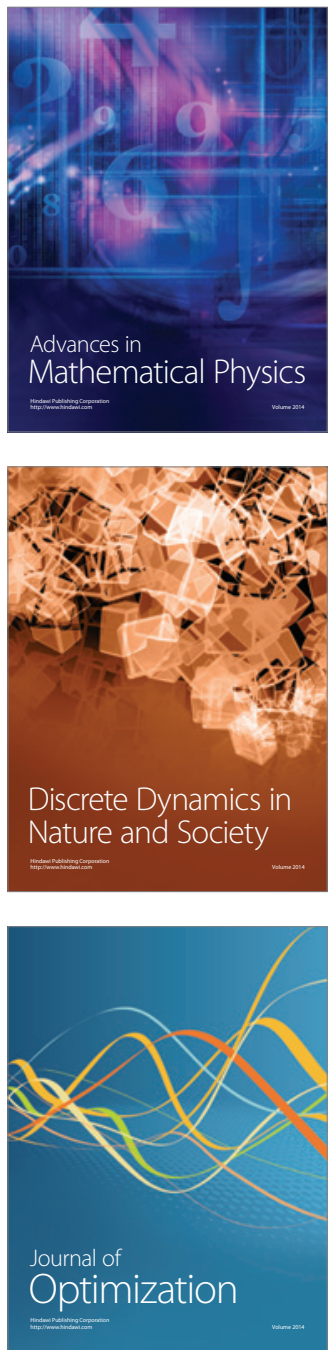